INPLASY

PROTOCOL

To cite: Mao et al. Catheter ablation vs. antiarrhythmic drugs as first-line therapy for symptomatic atrial fibrillation: network meta-analysis of randomized controlled trials. Inplasy protocol 202130098. doi:

10.37766/inplasy2021.3.0098

Received: 26 March 2021

Published: 27 March 2021

Corresponding author:

Hang Wang

15252336@qq.com

Author Affiliation:

First Affiliated Hospital of

Fujian Medical University

Support: None.

Review Stage at time of this submission: Piloting of the study selection process.

Conflicts of interest:

None declared.

\section{Catheter ablation vs. antiarrhythmic drugs as first-line therapy for symptomatic atrial fibrillation: network meta-analysis of randomized controlled trials}

Mao, Y1; Chen, X2; Huang, Q3; Yu, F4; Chen, J5; Wang, $\mathrm{H}^{6}$.

Review question / Objective: There have been studies comparing radiofrequency ablation (RFA) with antiarrhythmic drugs (AADs)/cryoballoon ablation (CBA) with AADs as the initial treatment strategy for paroxysmal atrial fibrillation (AF). However, it is not clear which of these two most commonly used ablation techniques is better as the first-line treatment strategy for paroxysmal AF.

Condition being studied: Atrial fibrillation.

Information sources: We will search the EMBASE, PubMed, and Cochrane Library databases for randomized controlled trials (RCTs) that compared CBA with AAD as first-line treatment for AF from the time of databases establishment up to March 2021.

INPLASY registration number: This protocol was registered with the International Platform of Registered Systematic Review and Meta-Analysis Protocols (INPLASY) on 27 March 2021 and was last updated on 27 March 2021 (registration number INPLASY202130098).

\section{INTRODUCTION}

Review question / Objective: There have been studies comparing radiofrequency ablation (RFA) with antiarrhythmic drugs (AADs)/cryoballoon ablation (CBA) with AADs as the initial treatment strategy for paroxysmal atrial fibrillation (AF). However, it is not clear which of these two most commonly used ablation techniques is better as the first-line treatment strategy for paroxysmal AF.

Condition being studied: Atrial fibrillation. 


\section{METHODS}

Participant or population: Adults with recurrent, non-permanent atrial fibrillation.

Intervention: Radiofrequency ablation, cryoballoon ablation.

Comparator: Class I or III antiarrhythmic drugs.

Study designs to be included: Randomized controlled trials.

Eligibility criteria: Adults with recurrent, non-permanent atrial fibrillation.

Information sources: We will search the EMBASE, PubMed, and Cochrane Library databases for randomized controlled trials (RCTs) that compared CBA with AAD as first-line treatment for $A F$ from the time of databases establishment up to March 2021.

Main outcome(s): Recurrence of any atrial tachyarrhythmia, defined according to each study's arrhythmia monitoring strategy.

Quality assessment / Risk of bias analysis: Two authors will independently assess studies for risk of bias using the Cochrane Risk of Bias tool.

Strategy of data synthesis: For a conventional pairwise meta-analysis, Risk ratio (RR) and $95 \%$ confidence interval $(\mathrm{Cl})$ was calculated to compare the effect of the combined therapy. The Cochrane 12 statistic was used to analyze the heterogeneity, which was divided into high ( $12 \geq 75 \%)$, moderate ( $12>25 \%$ and $<75 \%$ ), or low (12 $\leq 25 \%)$. When the heterogeneity was low, the fixed-effect model was used. Otherwise, use a random-effect model. $P$ value less than 0.05 was considered statistically significant. The RevMan software (version 5.3.5) was used. For indirect comparison, a Bayesian network meta-analysis was performed using $\mathbf{R}$ software. Markov chain Monte Carlo (MCMC) simulations were applied to estimate posterior probabilities with Gibbs sampling from at least 100,000 iterations. The probability of being the best treatment
(Prbest) was estimated and ranked for each intervention. The nodesplitting method was applied to assess network inconsistency.

Subgroup analysis: Perform subgroup analysis according to different ablation techniques (RFA vs. CBA).

Sensitivity analysis: We will attempt to explore the source of the heterogeneity by performing sensitivity analysis (stepwise exclusion of one study at a time).

Country(ies) involved: China.

Keywords: Cryoballoon ablation; Antiarrhythmic drugs; Radiofrequency ablation; Initial therapy; Atrial fibrillation.

Contributions of each author:

Author 1 - Yinjun Mao.

Author 2 - Xiaoying Chen.

Author 3 - Qunying Huang.

Author 4 - Fuling Yu.

Author 5 - Jianxing Chen.

Author 6 - Hang Wang. 